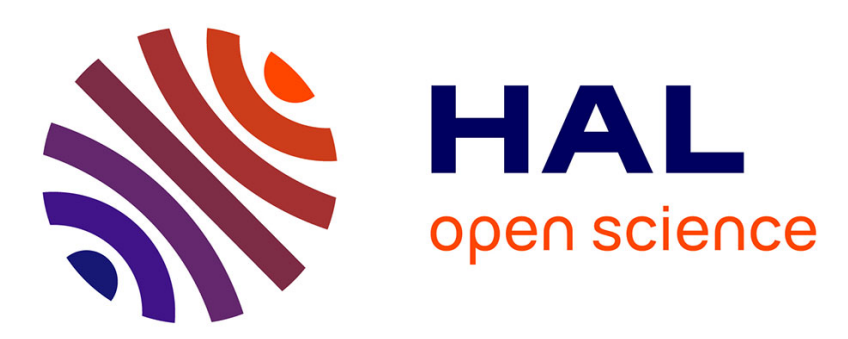

\title{
Performance analysis for tandem manufacturing lines under variable structure production control method.
} Konstantin Starkov, Alexander Pogromsky, J.E. Rooda

\section{To cite this version:}

Konstantin Starkov, Alexander Pogromsky, J.E. Rooda. Performance analysis for tandem manufacturing lines under variable structure production control method.. International Journal of Production Research, 2011, 10.1080/00207543.2011.564671 . hal-00715428

\section{HAL Id: hal-00715428 \\ https://hal.science/hal-00715428}

Submitted on 7 Jul 2012

HAL is a multi-disciplinary open access archive for the deposit and dissemination of scientific research documents, whether they are published or not. The documents may come from teaching and research institutions in France or abroad, or from public or private research centers.
L'archive ouverte pluridisciplinaire HAL, est destinée au dépôt et à la diffusion de documents scientifiques de niveau recherche, publiés ou non, émanant des établissements d'enseignement et de recherche français ou étrangers, des laboratoires publics ou privés. 


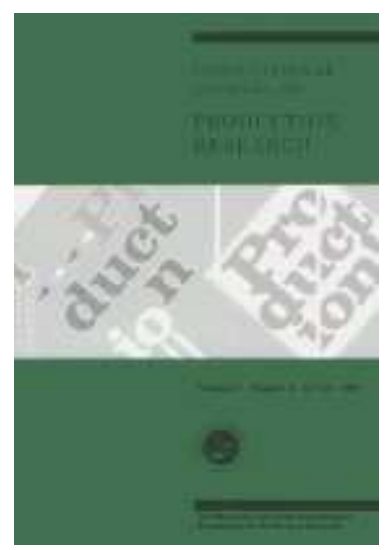

\section{Performance analysis for tandem manufacturing lines under variable structure production control method.}

\begin{tabular}{|r|l|}
\hline Journal: & International Journal of Production Research \\
\hline Manuscript ID: & TPRS-2010-IJPR-1210.R1 \\
\hline Manuscript Type: & Original Manuscript \\
\hline Author: & 18-Jan-2011 \\
\hline & $\begin{array}{l}\text { Complete List of Authors: } \\
\text { Mechanical Engineering } \\
\text { Rooda, J.E.; Eindhoven University of Technology, Mechanical } \\
\text { Engineering }\end{array}$ \\
\hline Keywords: & MANUFACTURING CONTROL SYSTEMS, PRODUCTION CONTROL \\
\hline Keywords (user): & \multicolumn{2}{|l}{} \\
\hline
\end{tabular}

\section{SCHOLARONE Manuscripts}


International Journal of Production Research

Vol. 00, No. 00, 00 Month 200x, 1-18

\title{
Performance analysis for tandem manufacturing lines under variable structure production control method
}

\author{
K.K. Starkov ${ }^{a *}$, A.Y. Pogromsky ${ }^{a}$ and J.E. Rooda ${ }^{a}$ \\ ${ }^{a}$ Department of Mechanical Engineering, Eindhoven University of Technology, \\ The Netherlands \\ (Received 00 Month 200x; final version received 00 Month 200x)
}

\begin{abstract}
In this paper authors introduce novel results on performance for surplus-based decentralized production control method. The main objective of this production method is to guarantee that the cumulative number of produced products follows the cumulative production demand on the output of any given network. As a starting point of our research a general idea of this method is presented for the case of one manufacturing machine. Then our analysis is extended to a line of $N$ machines. The proposed methodology is reformulated in terms of variable structure control. The production flow process is described by means of difference equations and in order to analyze performance Lyapunov theory approach is exploited. The obtained results on the production error bounds for each machine in the line and on the buffer content bounds for each intermediate buffer are discussed. Performance and robustness issues of the closed-loop flow line models are illustrated in numerical simulations. It is shown that the method can be implemented in the enterprise planning tools.
\end{abstract}

Keywords: manufacturing systems; tracking systems; discrete time systems; boundary conditions; variable structure control

\section{Introduction}

In the modern market keeping high competition in brands and varieties in type of products is the way for survival of manufacturing industries. Therefore production control methods with capabilities of quick responses to rapid changes in the demand and efficient distribution of the raw material throughout the network are of a big importance among leading manufacturers. Nowadays, the production control problem has been widely studied and a lot of valuable approaches including queuing theory, Petri nets, dynamic programming, linear programming, hybrid systems were proposed and some of them are implemented. Though up to this moment many methods have been developed, the factory performance remains unpredictable.

\footnotetext{
*Corresponding author. Email: k.starkov@tue.nl
} 
Some examples of implemented strategies are pull, push, and their combinations (see, e.g. Hopp and Spearman (2008), Deleersnyder et al. (1992), Ahn and Kaminsky (2005) and references therein). These control strategies, together with integrated computerbased systems, such as Manufacturing Resource Planning (MRP II), and Enterprise Resource Planning (ERP) (Vollmann et al. (2004)), are widely used by industries to control product flows in networks as well as inventory levels. Though these policies are conceptually simple, their response to disturbances and market fluctuations is not always fast enough for challenging requirements. Another often used control strategy is based on model predictive control (MPC) (see, e.g. Song et al. (2002), Doganis et al. (2007), Camacho and Bordons (2004) and references therein). This is a robust method that is capable of providing solutions for a production demand tracking problem. MPC is able to take into account hard constraints, such as the maximum production speed of the machine and buffer capacity restrictions. MPC is an effective method that can be used for real-time control of manufacturing systems, but it has two main drawbacks. The first one is that the optimization problem to be solved is generally very cumbersome, so it requires a lot of computational efforts, especially if the model presents some stochasticity in its behavior. The second one is that MPC strategy requires the knowledge of the future demand within a certain large enough finite control horizon. However, it is known that while dealing with demand planning the future is difficult to predict, even to the next time step. Thus, it may occur that the forecast presents certain inaccuracy which may result in production losses or backlog.

There is a substantial literature on control policies for manufacturing systems. Thus, many classifications of these policies are introduced by different authors. In this paper we will follow the classification introduced by S.R. Gershwin. In his work (Gershwin (2000)) the author separates the control policies in 3 main streams: token-based, time-based and surplus-based. In token-based approaches so called tokens are generated and utilized in order to trigger certain events occurring in the manufacturing system. The most famous examples of such a policy are Kanban (Rees et al. (1987)), Conwip (Spearman et al. (1990), Ioannidis and Kouikoglou (2008)) and Basestock (Silver et al. (1998)). In timebased approaches the control decisions depend on a time when a certain operation should take place, i.e. Material Resurce Planning, Least Stack and Earliest Due Date strategies (see, e.g., Burgess and Passino (1997)). In the surplus approach control decisions are made based on the production error which is the difference between the cumulative demand and the cumulative output of the system. Some references for these strategies are presented later in this section.

In this paper we tackle the problem of performance analysis (see, e.g., Ruifeng and Subramaniam (2010)) for a surplus-based approach in control of manufacturing networks within the scope of demand driven manufacturing control problems. Each machine in the manufacturing system coordinates its individual production with those of the rest of the system. Its primary objective may be viewed as the manufacturing of sufficient quantity of parts to satisfy the demand of its immediate downstream machine and some desired amount for a purpose of back-up material storage in its downstream buffer. The proposed methodology is reformulated in terms of variable structure control. The production flow process is described by means of difference equations and in order to analyse performance Lyapunov theory approach is exploited (see, e.g., Dashkovskiy et al. (2010), Scholz-Reiter et al. (2010) and references there in).

The novelty of our results, concerning surplus-based approach (see, e.g. Bielecki and Kumar (1988), Bonvik et al. (1997), Lefebvre (1999), Gershwin (2000), Quintana (2002), Kogan and Perkins (2003), Boukas (2006), Stockton 
et al. (2007), Subramaniam et al. (2009), Savkin and Somlo (2009), Nilakan$\tan (2010)$ and references there in), can be summarized as follows. The proposed tandem production model is considered in discrete time. The production speed of each machine is defined as deterministic with bounded perturbations. The future production demand is assumed to be unknown and with bounded fluctuations. As a result, strict, so called "worst case" bounds on a content of intermediate buffers and production tracking errors for one machine and for a unidirectional tandem production line are obtained as a starting point of our research. In order to monitor a current market situation, and the current inventory level of given network the proposed strategy can be easily combined with integrated computer-based systems (such as previously mentioned in this section).

In particular, this paper contains an explicit description of flow models (see, e.g. Dallery and Gershwin (1992), Alvarez-Vargas et al. (1994), Pogromsky et al. (2009) and references therein) for one machine and a line of manufacturing machines. Here a variable structure control (see, e.g. Utkin (1983), Khalil (2002)) is introduced as a control technique in order to give a solution to the demand tracking problem. Special attention is paid to the constraints presented in the network, such as capacity and buffer limitations. Each machine in the network can produce a restricted number of products in a fixed period of time, known as the capacity constraint. The content of the buffer between two machines is given by the difference between the total number of products produced by the upstream machine and the total number of products produced by the downstream machine. Considering that a manufacturing line has a unidirectional product flow implies that the buffer content can never be negative, e.g. the downstream machine cannot produce more than the upstream one.

The paper is organized as follows. First, in Section 2 the flow model of one manufacturing machine with variable structure control is presented, where the analysis of production error trajectories is developed. Then the flow model of a manufacturing line with variable structure control is analyzed in Section 3. Here necessary conditions are derived to guarantee the uniform ultimate boundedness of the production error trajectories of each machine. Performance and robustness issues of the closed-loop flow models are illustrated in numerical simulations in Section 4. Finally, Section 5 contains conclusions and implementation in a real environment.

\section{One Machine}

\subsection{Flow Model}

In discrete time a cumulative number of produced products in time $k$ for a simple manufacturing machine can be described as a sum of its production rates at each time step till time $k$. Thus the flow model of one manufacturing machine in discrete time is defined as

$$
y(k+1)=y(k)+u(k)+f(k)
$$

where $y(k) \in \mathbb{R}$ is the cumulative output of the machine in time $k, u(k) \in \mathbb{R}$ is the control signal, and $f(k) \in \mathbb{R}$ is an unknown external disturbance. Under the assumption that there is always sufficient quantity of the raw material to feed the machine, the control aim is to track the non-decreasing cumulative production demand. We define the production 
demand by using $y_{d}(k) \in \mathbb{R}$ given by

$$
y_{d}(k)=y_{d 0}+v_{d} k+\varphi(k)
$$

where $y_{d 0}$ is a positive constant that represents the initial production demand, $v_{d}$ is a positive constant that defines the average desired demand rate, and $\varphi(k) \in \mathbb{R}$ is the bounded fluctuation that is imposed on the linear demand $v_{d} k$. In order to give a solution to this tracking problem the following control input

$$
u(k)=\mu \operatorname{sign}_{+}(\varepsilon(k))
$$

is considered. Here $\mu$ is a positive constant that represents the processing speed of the machine, step function $\operatorname{sign}_{+}(\varepsilon(k))=(1$, if $\varepsilon(k)>0 \mid 0$, otherwise), and $\varepsilon(k) \in \mathbb{R}$ is the output production error with respect to the demand. This production error is given by $\varepsilon(k)=y_{d}(k)-y(k)$, where $\left.\varepsilon(k+1)\right)-\varepsilon(k)$ along the solutions of $\varepsilon(k)$ is given by:

$$
\varepsilon(k+1)-\varepsilon(k)=v_{d}+\Delta \varphi(k)-\mu \operatorname{sign}_{+}(\varepsilon(k))-f(k),
$$

with $\Delta \varphi(k)=\varphi(k+1)-\varphi(k)$. It is followed from (4) that in order to guarantee a proper demand trajectory tracking the product demand cannot be higher than the machine processing speed, which in this case is $\mu$ lots per time unit. Thus, let us consider that all machine perturbations $W(k)=\Delta \varphi(k)-f(k)$ from (4) are bounded by

$$
\alpha_{1}<W(k)<\alpha_{2}, \quad \forall k \in \mathbb{N},
$$

where $\alpha_{1}, \alpha_{2}$ are some constants that satisfy

$$
\begin{aligned}
& \alpha_{2}<\mu-v_{d}, \\
& \alpha_{1}>-v_{d} .
\end{aligned}
$$

By (6) and (7) we state that the machine can never produce products faster than its maximal speed and that considering the presence of perturbations bounded by $\left(\alpha_{1}, \alpha_{2}\right)$ the demand rate can only de positive, respectively. Thus, from (5), (6), and (7) the following condition (also known as capacity condition) holds

$$
0<v_{d}+W(k)<\mu \text {. }
$$

Basically, in order to follow the product demand, variable structure controller $\mu \operatorname{sign}_{+}(\varepsilon(k))$ is included in the flow model of one machine. The production error of a single machine is defined as the difference between the cumulative demand and the cumulative number of products produced up to this moment.

\subsection{Production error analysis}

In this section we analyse the tracking error trajectories behavior of the flow model (4). 
Theorem 2.1: Assume that the discrete time system defined by (1) with control input (3) satisfies condition (8). Then all solutions of (4) are uniformly ultimately bounded by

$$
\begin{aligned}
& \limsup _{k \rightarrow \infty} \varepsilon(k) \leq v_{d}+\alpha_{2}, \\
& \liminf _{k \rightarrow \infty} \varepsilon(k) \geq v_{d}+\alpha_{1}-\mu .
\end{aligned}
$$

Proof: The proof of Theorem 2.1 is provided in the first part of the proof of Theorem 3.4 .

\section{A Line of Machines}

\subsection{Flow Model}

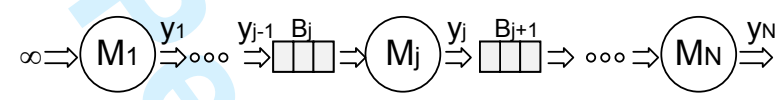

Figure 1. Schematics of a line of $N$ manufacturing machines.

The flow model of a manufacturing line is presented in this section. Figure 1 presents a schematics of a line of $N$ manufacturing machines with machines $M_{j}$, buffers $B_{j}$, and infinite product supply. Here the control strategy for one machine is modified with respect to the number of buffers and machines present in the line. A new limitation such as desired buffer content is considered in the model.

The flow model of the manufacturing line is defined as

$$
\begin{aligned}
y_{1}(k+1)= & y_{1}(k)+\beta_{1}(k), \\
y_{j}(k+1)= & y_{j}(k)+\left(\beta_{j}(k)\right) \operatorname{sign}_{B u f f}\left(w_{j}(k)-\beta_{j}(k)\right), \\
& \forall j=2, \ldots, N,
\end{aligned}
$$

where $y_{j}(k)$ is the cumulative output of machine $M_{j}$ in time $k, w_{j}(k)=y_{j-1}(k)-y_{j}(k)$ is the buffer content of buffer $B_{j}, \beta_{j}(k)=u_{j}(k)+f_{j}(k), \forall j=1, \ldots, N, f_{j}$ is the external disturbance affecting machine $M_{j}$ (e.g. production speed variations, undesired delay), $u_{j}$ is the control input of machine $M_{j}$ and $\operatorname{sign}_{B u f f}\left(w_{j}(k)-\beta_{j}(k)\right)=\left(1\right.$, if $w_{j}(k)-\beta_{j}(k) \geq 0 \mid 0$, otherwise).

In order to give a solution to the demand tracking problem we consider the following control inputs:

$$
\begin{aligned}
u_{j}(k)= & \mu_{j} \operatorname{sign}_{+}\left(\varepsilon_{j+1}(k)+\left(w_{d_{j+1}}-w_{j+1}(k)\right)\right) \\
& \forall j=1, \ldots, N-1 \\
u_{N}(k)= & \mu_{N} \operatorname{sign}_{+}\left(y_{d}(k)-y_{N}(k)\right),
\end{aligned}
$$

where $\mu_{j}$ is the processing speed of machine $j, w_{d_{j+1}}$ is the desirable buffer level of buffer $B_{j+1}$ and $\varepsilon_{j+1}$ is the tracking error of machine $M_{j+1}$. The tracking error of each machine 
is given by:

$$
\begin{aligned}
\varepsilon_{j}(k)= & \varepsilon_{j+1}(k)+\left(w_{d_{j+1}}-w_{j+1}(k)\right) \\
& \forall j=1, \ldots, N-2 \\
\varepsilon_{N-1}(k)= & \varepsilon_{N}(k)+\left(w_{d_{N}}-w_{N}(k)\right) \\
\varepsilon_{N}(k)= & y_{d}(k)-y_{N}(k) .
\end{aligned}
$$

It follows from (17) that the error of machine $M_{N}$ is defined exactly as for the single machine case. The buffer restriction, as seen from (12), is the only difference in the flow model of machine $M_{N}$ with the flow model of (1). For (15), (16) new considerations are applied for the tracking error of each machine $M_{j}$, where $j=1, \ldots, N-1$. Thus, tracking error $\varepsilon_{j}(k)$ depends on the number of produced products $y_{j}(k)$ with respect to current demand $y_{d}(k)$ and desired buffer content $w_{d_{j+1}}$ of each downstream buffer. This means that every upstream machine needs to supply $w_{d_{j+1}}$ lots more than the downstream one. Constant parameter $w_{d}$ is introduced in order to prevent downstream machines from lot starvation, e.g. in case of a sudden growth of the product demand.

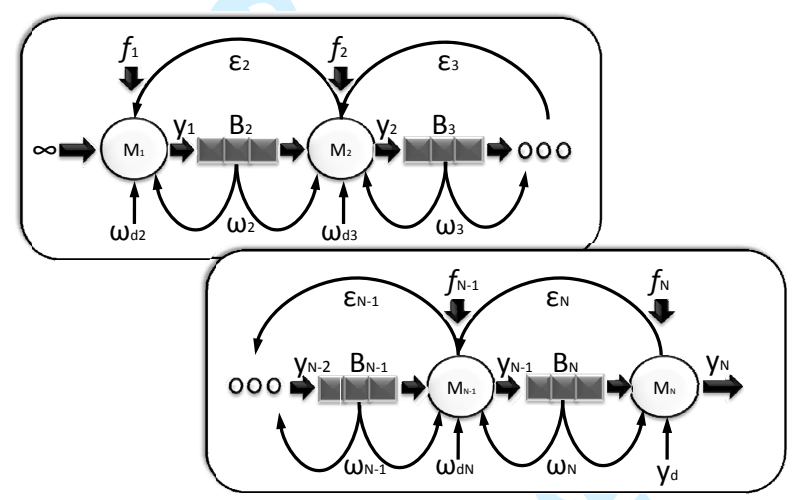

Figure 2. Flow model diagram for a line of $\mathrm{N}$ manufacturing machines.

Basically, model (11), (12) describes the product flow through the line of $N$ manufacturing machines (Fig.2). The first machine described by (11) is considered to have always an access to the raw material and the raw material is always sufficient. The administration of this raw material to machine $M_{1}$ is decided by the control input (13). Here we consider that our control input is acting as an authorizing switch, which turns on $M_{1}$ if its tracking error (15) is positive and turns $M_{1}$ off if its tracking error is negative or zero. Tracking error $\varepsilon_{1}(k)$, see (15), consists of the difference between what is done $\left(w_{2}(k)\right)$, what have to be done $\left(\varepsilon_{2}(k)\right)$ and what have to be always in the buffer $\left(w_{d_{2}}\right)$. It can be seen from (15), (16) and (13) that the same tracking error and control logics were applied for the rest of the machines till machine $M_{N-1}$. As for the last machine in the line, which is machine $M_{N}$ (Fig.2), the control action is still based on the authorizing switch. However, the difference consists in the logic that triggers this switch. We expect that on the output of machine $M_{N}$ the cumulative product demand is followed by cumulative production of this machine. The control switch activates or deactivates the machine based directly on the production demand status (17). This control logic is the same as 
in the one machine case presented in the previous section. The substantial difference in models of the rest of machines from machine one can be appreciated through the general flow model (12). Here for each machine in the line we introduce an extra restriction on the buffer content of each upstream buffer. Function $\operatorname{sign}_{B u f f}\left(w_{j}(k)-\beta_{j}(k)\right)$ is acting as an extra authorization together with the control input. Thus, any machine $M_{j}$, with $j=2, \ldots, N$, is activated only if two authorizations are given. The first authorization comes from control input $\left(u_{j}(k)\right)$ of the machine, which is based on the current tracking error status of this machine $\left(\varepsilon_{j}(k)\right)$. The second authorization comes from the buffer content restriction which is granted if the buffer contains at least the minimal number of products required $\left(\beta_{j}(k)\right)$ in order for the machine $M_{j}$ to start its work. It is also important to take into account that the control actions are decentralized throughout the network. In other words the control action of each machine in the line depends only on the tracking error of its neighboring downstream machine (except for machine $M_{N}$, which depends directly on cumulative demand input) and the current buffer content of its upstream buffer (Fig.2). This gives our flow model an extra robustness with respect to the undesired events such as temporal machine setup or breakdown.

For further analysis, let us rewrite flow model (11), (12) in a closed-loop with (13), (14) as

$$
\begin{aligned}
& \Delta \varepsilon_{1}(k)=v_{d}+\Delta \varphi(k)-f_{1}(k)-\mu_{1} \operatorname{sign}_{+}\left(\varepsilon_{1}(k)\right), \\
& \Delta \varepsilon_{j}(k)=v_{d}+\Delta \varphi(k)-f_{j}(k)-\mu_{j} \operatorname{sign}_{+}\left(\varepsilon_{j}(k)\right) \operatorname{sign}_{B u f f}\left(w_{j}(k)-\beta_{j}(k)\right),
\end{aligned}
$$

where $\Delta \varepsilon_{j}(k)=\varepsilon_{j}(k+1)-\varepsilon_{j}(k)$ and $W_{j}(k)=\Delta \varphi(k)-f_{j}(k), \forall j=1, \ldots, N$.

Here we consider that system (18), (19) satisfies the following assumptions.

Assumption 3.1: (Boundedness of perturbations) There are constants $\alpha_{1}, \alpha_{2}$ and $\alpha_{3}$ such that $W_{j}(k)=\Delta \varphi(k)-f_{j}(k)$ satisfies

$$
\alpha_{1}<W_{j}(k)<\alpha_{2}, \forall k \in \mathbb{N}, j=1, \ldots, N
$$

and $f_{j}(k)$ satisfies

$$
f_{j}(k) \leq \alpha_{3}, \forall k \in \mathbb{N}, j=1, \ldots, N
$$

Assumption 3.2: (Capacity condition) Constants $\alpha_{1}, \alpha_{2}$ satisfy the following inequalities

$$
\begin{aligned}
& \alpha_{2}<\mu_{j}-v_{d}, \forall j=1, \ldots, N, \\
& \alpha_{1}>-v_{d} .
\end{aligned}
$$

Thus, from (20), (22), and (23) the following condition holds

$$
0<v_{d}+W_{j}(k)<\mu_{j}, \forall j=1, \ldots, N
$$

It is important to notice that each $M_{j}$ machine in the line has a processing speed of $\mu_{j}$ lots per time unit, which can differ from the rest of the machines, and the buffer condition is considered as 


$$
w_{j}(k) \geq \beta_{j}(k), \forall j=2, \ldots, N \text {. }
$$

Thus, from (15), (16) and (25) the following tracking error condition holds

$$
\varepsilon_{j}(k) \geq \beta_{j}(k)-w_{d_{j}}+\varepsilon_{j-1}(k), \forall j=2, \ldots, N,
$$

where $w_{d_{j}}$ satisfy the following assumption.

Assumption 3.3: (Desired buffer content condition) The constants $w_{d_{j}}$ comply with the following inequality

$$
w_{d_{j}} \geq \mu_{j}+\mu_{j-1}+\alpha_{3}+\alpha_{2}-\alpha_{1}
$$

from where it follows that

$$
w_{d_{j}} \geq \beta_{j}(k)+\mu_{j-1}+\alpha_{2}-\alpha_{1}, k \in \mathbb{N}, j=2, \ldots, N
$$

If the condition (25) is not satisfied then

$$
\varepsilon_{j-1}(k) \stackrel{(15,16,26)}{>} \mu_{j-1}+\alpha_{2}-\alpha_{1}+\varepsilon_{j}(k), \forall j=2, \ldots, N .
$$

\subsection{Production Error Analysis}

In this section we present results respecting the production error trajectories behavior of the flow model (18), (19).

Theorem 3.4: Assume that the discrete time system defined by (18), (19) satisfies Assumptions 3.1, 3.2, and 3.3. Then all solutions of (18) and (19) are uniformly ultimately bounded by

$$
\begin{aligned}
& \limsup _{k \rightarrow \infty} \varepsilon_{j}(k) \leq v_{d}+\alpha_{2}, \\
& \liminf _{k \rightarrow \infty} \varepsilon_{j}(k) \geq v_{d}+\alpha_{1}-\mu_{j} .
\end{aligned}
$$

Proof: The proof of Theorem 3.4 is provided in Appendix A.

In consequence, for the buffer content $w_{j}(k)$ of each buffer $B_{j}$ defined by (15), (16), considering the obtained tracking error bounds (28), (29), it holds that

$$
\limsup _{k \rightarrow \infty} w_{j}(k) \leq \mu_{j-1}+\alpha_{2}-\alpha_{1}+w_{d_{j}} .
$$

Now, in order to support the present development let us extend our analysis to simulation results. 


\section{Simulation Results}

\subsection{One machine case}

Simulation results for one manufacturing machine, driven by the variable structure regulator (3), are presented in this section. Here the processing speed was fixed to 5 lots per time unit.

Results from Figure 3 show that output $y(k)$ follows product demand $y_{d}(k)$ with tracking error $\varepsilon(k)$ bounded by $4 \geq \varepsilon(k) \geq 0$ lots, which satisfies (9), (10). Here, initial conditions were set to $y_{d 0}=20, y(0)=0$. Market fluctuations as well as external perturbations were set to the zero value. The tracking error from Figure 3 (b) is generated in this form due to the machine production speed limit of 5 lots per time unit with demand increment $\Delta y_{d}(k)$ of 4 lots per time unit. This means that after the moment when the steady state is reached (20 time steps) demand signal $y_{d}(k)$ is 4 lots higher than output $y(k)$. In this case the control responds with authorization for 5 lots, which are generated in the next time step, where the cumulative demand grows in another 4 lots. Thus, the current output is 3 lots lower than the current demand. Then for the next 4 time steps the machine keeps producing lots until the demand is reached again at time step 25 with $y_{d}(k)$ of 120 lots. Now during next time step the machine remains idle while the demand grows to 124 lots. Thus, the sequence from the 20th time step to the 25 th is repeated.

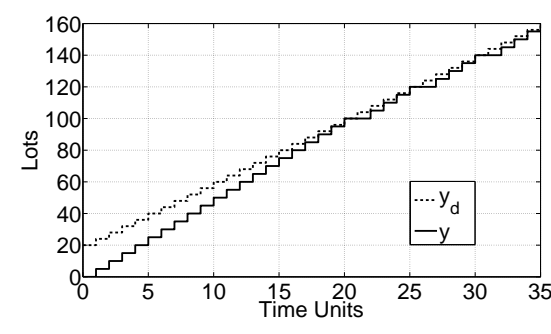

(a)

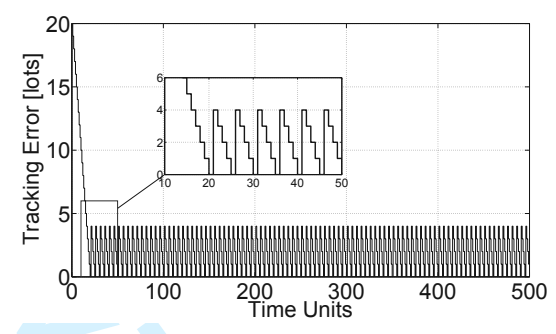

(b)

Figure 3. Demand vs Output (a) and Tracking Error (b), with $v_{d}=4$ and $y_{d 0}=20$.

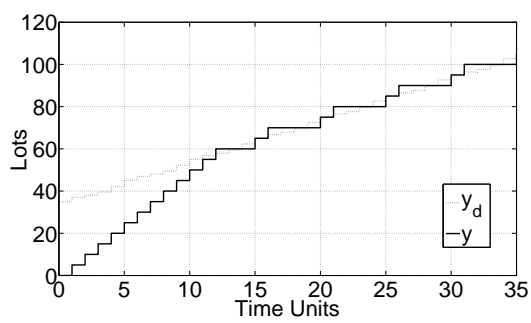

(a)

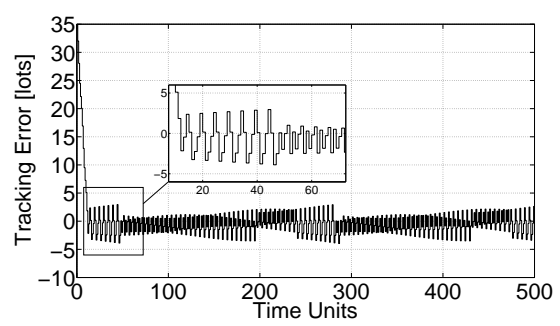

(b)

Figure 4. Demand vs Output (a) and Tracking Error (b), with $y_{d 0}=35, v_{d}=2$ and $\varphi(k)=\sin (5 k)$.

Figure 4(b) shows the tracking error and the output response of the machine to the nonlinear demand growth. Here product demand $y_{d}(k)=35+2 k+\sin (5 k)$. The resulting tracking error is bounded by $3>\varepsilon(k)>-4$ lots, which satisfies (9) and (10). It can be 
observed from Figure 4(a) that for given initial demand of 35 lots and initial output of 0 lots the machine manages to reach the demand trajectory in 12 time steps.

Finally we conclude that simulation results reflect the expected flow model behavior for all the product demands which are given in this section. These product demands were selected in order to test the model behavior inside the boundary of the given capacity condition (8).

\subsection{Four machines case}

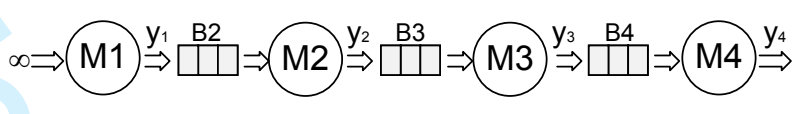

Figure 5. Schematics of a line of 4 manufacturing machines.

Simulation results for a line of 4 manufacturing machines (Fig. 5), driven by variable structure regulators (13), and (14) are presented in this section. For all the examples processing speed for each machine was set to $\mu_{j}=<8,10,7,6>$ (lots per time unit), with $j=1, . ., 4$, and the desired buffer content of each buffer was selected considering (26) as $w_{d_{j}}=<20,18,14>$ (lots), with $j=2, . ., 4$.

The tracking error of each machine in the line is depicted in Figure 7. Here the initial conditions $\left(y_{d 0}, y_{1}(0), y_{2}(0), y_{3}(0), y_{4}(0)\right)$ were set to the zero value. After the first 24 time steps, as it is shown in Figures 6 (a) and 7, the system reaches its steady state. Tracking errors are maintained inside $[-2,5]$ lots for machine $M_{1},[-4,1]$ lots for machine $M_{2},[-1,5]$ lots for machine $M_{3}$, and [0,5] lots for machine $M_{4}$, which satisfy (28) and (29). From Figure 6(b) it can be observed that the inventory level of each buffer satisfies the upper bound restriction (30).

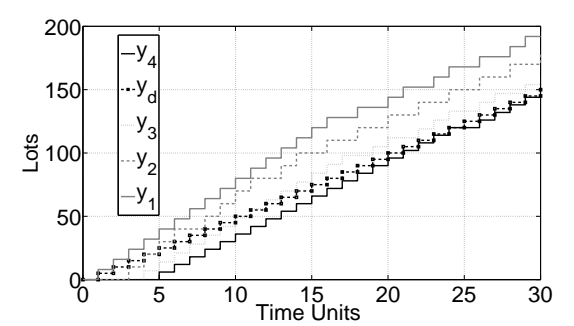

(a)

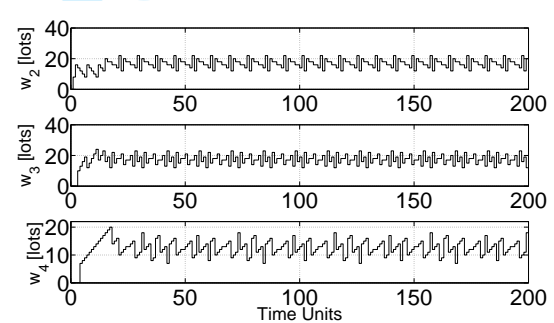

(b)

Figure 6. Outputs $y_{j}(k)$ vs. Demand $y d(k)(\mathrm{a})$ and Buffer Content $w_{j}(k)(\mathrm{b})$, with $v_{d}=5$.

Further, the output response and the tracking error of each machine are depicted in Figures 8 and 9 (a) for the same initial conditions as in the previous example. Here it is noticeable that after the first 7 time steps the output of machine $M_{4}$ reaches the current demand trajectory. Tracking errors are maintained inside [-6,4] lots for machine $M_{1}$, [-8,4] lots for machine $M_{2},[-5,4]$ lots for machine $M_{3}$, and [-4,4] lots for machine $M_{4}$, which satisfy (28), (29). The inventory level of each buffer is depicted in Figure 9(b). We indicate that the buffer content of each buffer satisfies the upper bound restriction (30), if given desired inventory level of each buffer satisfies (26). 
7

8

9

10

11

12

13

14

15

16

17

18

19

20

21

22

23

24

25

26

27

28

29

30

31

32

33

34

35

36

37

38

39

40

41

42

43

44

45

46

47

48

49

50

51

52

53

54

55

56

57

58

59

60
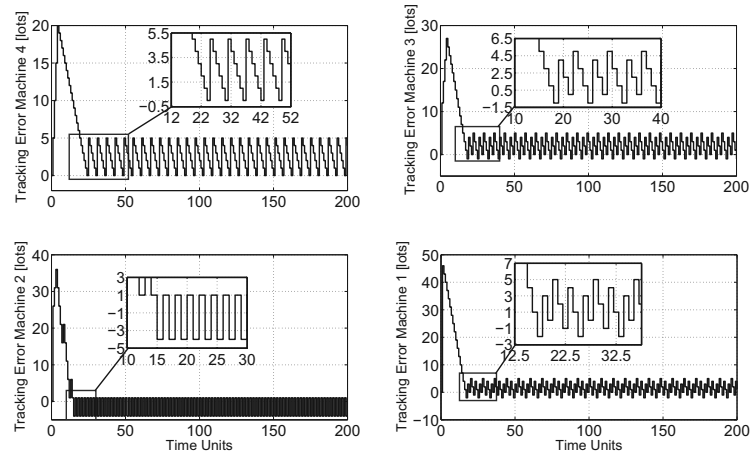

Figure 7. Tracking Error $\varepsilon_{j}(k)$, with $v_{d}=5$.
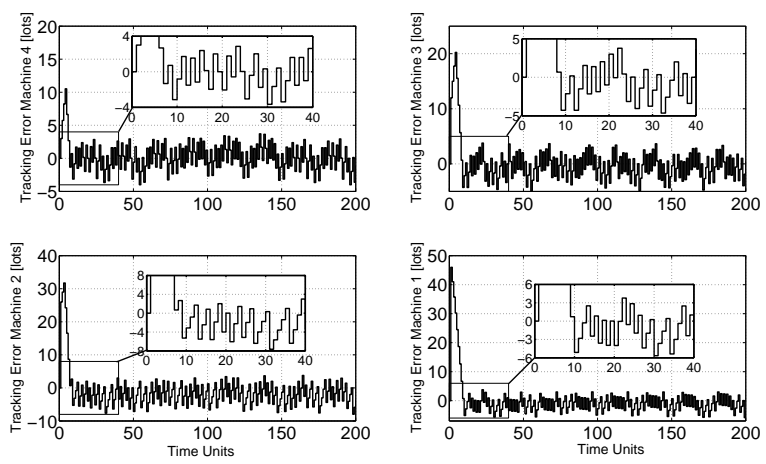

Figure 8. Tracking Error $\varepsilon_{4}(k)$, with $v_{d}=3$ and $\Delta \varphi(k)=\sin (50 k)$.

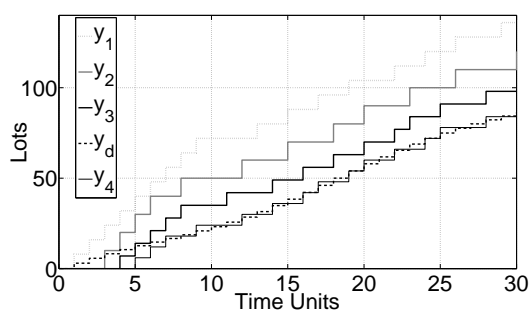

(a)

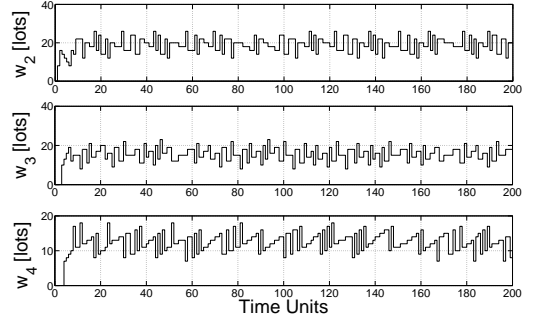

(b)

Figure 9. Outputs $y_{j}(k)$ vs. Demand $y d(k)$ (a) and Buffer Content $w_{j}(k)(\mathrm{b})$, with $v_{d}=3$ and $\Delta \varphi(k)=\sin (50 k)$.

Finally, presented simulation results on selected examples reflect the desired flow model behavior. All technical conditions proposed in this section corresponds to analytical results described in Section 3. 


\section{Conclusion}

The performances of a tandem production line under variable structure surplus control has been studied. Developed results prove the uniform ultimate boundedness for trajectories of each tracking error and provide the upper bound for each buffer content in a line of machines where each machine has a variable processing. Various simulation results are presented and discussed in order to illustrate and support analytical results. Simulation results reflect effectiveness and robustness of the flow models.

As can be seen from the model description, the control algorithm requires to have a knowledge of external and internal variables, which affect the production line functionality, as well as performance specifications of each machine. The external variable which is the current production demand $y d(k)$ as well as internal variables which are buffer contents $w_{j}(k)$ of each buffer can be obtained from existing planning details of a given company. Performance specifications such as machine production speed can be deduced from the experimental data or obtained from the user specifications of a given machine. Thus having this knowledge the production method can be implemented in form of an algorithm as a manufacturing line control tool. Further, if the conditions from Theorem 3.4 are satisfied then the system's results on performance can be directly obtained from formulas (28), (29) and (30). This can also significantly reduce the computational time needed for an order release planning.

Furthermore, studies on variable structure control policy's application to re-entrant network, multiple part type production systems, and performance analysis with the presence of production delays and setup times as well as it comparison with another surplus based strategies will be pursued in our future research.

\section{Acknowledgement}

The research leading to these results has received partial funding from the European Community's Seventh Framework Programme (FP7/2007-2013) under grant agreement no. INFSO-ICT-223844.

\section{References}

Ahn, H. and Kaminsky, P., 2005. Production and distribution policy in a two-stage stochastic push-pull supply chain. IIE Transactions, 37 (7), 609-621.

Alvarez-Vargas, R., Dallery, Y., and David, R., 1994. A study of the continuous flow model of production lines with unreliable machines and finite buffers. Journal of Manufacturing Systems, 13 (3), 221-234.

Bielecki, T. and Kumar, P., 1988. Optimality of zero-inventory policies for unreliable manufacturing systems. Operations Research, 36 (4), 532-541.

Bonvik, A., Couch, C., and Gershwin, S., 1997. A comparison of production-line control mechanisms. International Journal of Production Research, 35 (3), 789-804.

Boukas, E., 2006. Manufacturing Systems: LMI Approach. IEEE Transactions on Automatic Control, 51 (6), 1014-1018.

Burgess, K. and Passino, K., 1997. Stable scheduling policies for flexible manufacturing systems. IEEE Transactions on Automatic Control, 42 (3), 420-425. 
Camacho, E.F. and Bordons, C., 2004. Model Predictive Control. 2nd London: SpringerVerlag.

Dallery, Y. and Gershwin, S., 1992. Manufacturing flow line systems: a review of models and analytical results. Queueing Systems, 12, 3-94.

Dashkovskiy, S., Karimi, H., and Kosmykov, M., 2010. A LyapunovRazumikhin approach for stability analysis of logistics networks with time-delays. International Journal of Systems Science Online.

Deleersnyder, J., et al., 1992. Integrating Kanban type PULL systems and MRP type PUSH systems: insights from a Markovian Model. IIE Transactions, 24 (3), 43-56.

Doganis, P., Aggelogiannaki, E., and Sarimveis., H., 2007. A Model Predictive Control and Time Series Forecasting Framework for Supply Chain Management. Journal of Applied Mathematics and Computer Sciences, 3, 139-143.

Gershwin, S., 2000. Design and operation of manufacturing systems: the control-point policy. IIE Transactions, 32, 891-906.

Hopp, W.J. and Spearman, M.L., 2008. Factory physics. 3nd McGraw-Hill.

Ioannidis, S. and Kouikoglou, V., 2008. Revenue management in single-stage CONWIP production systems. International Journal of Production Research, 46 (22), 65136532.

Khalil, H.K., 2002. Nonlinear Systems. 3rd Prentice-Hall.

Kogan, K. and Perkins, J., 2003. Infinite horizon production planning with periodic demand: solvable cases and a general numerical approach. IIE Transactions, 35 (1), $61-71$.

Lefebvre, D., 1999. Feedback control designs for manufacturing systems modelled by continuous Petri nets. International Journal of Systems Science, 30 (6), 591-600.

Nilakantan, K., 2010. Enhancing supply chain performance with improved order-control policies. International Journal of Systems Science, 41 (9), 1099-1113.

Pogromsky, A., et al., 2009. Decentralized feedback control of manufacturing machines. In: 35th Conference of the IEEE Industrial Electronics Society (IECON) CD-ROM, Porto, Portugal.

Quintana, R., 2002. Recursive linear control of order release to manufacturing cells with random yield. IIE Transactions, 34, 489-500.

Rees, L., et al., 1987. Dynamically adjusting the number of Kanbans in a Just-in-time production system using estimated values of leadtime. IIE Transactions, 19, 199207.

Ruifeng, C. and Subramaniam, V., 2010. Performance evaluation for tandem multifactory supply chains: an approximate solution. International Journal of Production Research Online.

Savkin, A. and Somlo, J., 2009. Optimal distributed real-time scheduling of flexible manufacturing networks modeled as hybrid dynamical systems. Robotics and ComputerIntegrated Manufacturing, 25, 597 - 609.

Scholz-Reiter, B., et al., 2010. Stability analysis of autonomously controlled production networks. International Journal of Production Research.

Silver, E., Pyke, D., and Peterson, R., 1998. Inventory management and production planning and scheduling. 3rd John Wiley \& Sons: New York, NY.

Song, K., et al., 2002. Partial least square-based model predictive control for large-scale manufacturing processes. IIE Transactions, 34 (10), 881-890.

Spearman, M., Woodruff, D., and Hopp, W., 1990. CONWIP. A pull alternative to kanban. International Journal of Production Research, 28, 879-894.

Stockton, D., Ardon-Finch, J., and Khalil, R., 2007. Control point policy: part 1 effi- 
ciency within make-to-order environments. International Journal of Production Research, 46 (11), 2927-2943.

Subramaniam, V., et al., 2009. A WIP control policy for tandem lines. International Journal of Production Research, 47 (4), 1127-1149.

Utkin, V., 1983. Variable Structure Systems: Present and Future. Automation and Remote Control, 44, 1105-1120.

Vollmann, T.E., Berry, W.L., and Whybark, D.C., 2004. Manufacturing Planning and Control Systems for Supply Chain Management. 5th McGraw-Hill.

\section{Appendix A. Proof of Theorem 3.4}

Let us prove that Theorem 3.4 holds for a line of 2 manufacturing machines $(j=1,2)$ defined by (18) and (19). With this goal, let us introduce the following Lyapunov function

$$
V^{2 M}\left(\varepsilon_{1}, \varepsilon_{2}\right)=\max \left\{V_{1}\left(\varepsilon_{1}\right), V_{2}\left(\varepsilon_{2}\right)\right\}
$$

where

$$
\begin{aligned}
V_{j}\left(\varepsilon_{j}\right)= & \max \left\{-\varepsilon_{j}-\mu_{j}+v_{d}+\alpha_{1}, \varepsilon_{j}-v_{d}-\alpha_{2}, 0\right\}>0, \\
& \forall \varepsilon_{j} \notin\left[v_{d}+\alpha_{1}-\mu_{j}, v_{d}+\alpha_{2}\right], j=1,2 .
\end{aligned}
$$

Here for the sake of brevity $V^{2 M}\left(\varepsilon_{1}(k), \varepsilon_{2}(k)\right)=V_{k}^{2 M}, V_{j}\left(\varepsilon_{j}(k)\right)=V_{j, k}$, with $V^{2 M}=0$ $\forall \varepsilon_{j} \in\left[v_{d}+\alpha_{1}-\mu_{j}, v_{d}+\alpha_{2}\right]$.

Thus, $\Delta V_{k}^{2 M}$ along the solutions of $\varepsilon_{1}(k)$ and $\varepsilon_{2}(k)$ is given by

$$
\Delta V_{k}^{2 M}=V_{k+1}^{2 M}-V_{k}^{2 M}=\max \left\{V_{1, k+1}, V_{2, k+1}\right\}+\min \left\{-V_{1, k},-V_{2, k}\right\},
$$

where

$$
V_{j, k+1}=\max \left\{\begin{array}{c}
-\varepsilon_{j}(k)-W_{j}(k)+\alpha_{1}-\mu_{j}+\mu_{j} \eta_{j, k}, \\
\varepsilon_{j}(k)+W_{j}(k)-\alpha_{2}-\mu_{j} \eta_{j, k}, \\
0
\end{array}\right\}
$$

Here for the sake of brevity we introduce $\eta_{j, k}$ as

$$
\begin{aligned}
& \eta_{1, k}=\operatorname{sign}_{+}\left(\varepsilon_{1}(k)\right), \\
& \eta_{2, k}=\operatorname{sign}_{+}\left(\varepsilon_{2}(k)\right) \operatorname{sign}_{B u f f}\left(w_{2}(k)-\beta_{2}(k)\right) .
\end{aligned}
$$

In order to perform a more detailed analysis on $\Delta V_{k}^{2 M}$, let us divide this proof into 2 cases.

Case 1 (Sufficient Buffer Content)

Suppose that $w_{2}(k)$ satisfies the following inequality

$$
w_{2}(k) \geq \beta_{2}(k), \forall k \in \mathbb{N}
$$


which means that machine $M_{2}$ has sufficient material in its buffer $B_{2}$ in order to start working and machine $M_{1}$ always has an access to the infinite raw material supply. Thus these machines have an independent behavior and it will be sufficient to analyse the increment of only one of the functions $V_{j, k}$ in order to determine the behavior of $\Delta V_{k}^{2 M}$.

Let us assume that $\varepsilon_{j}(k)$ satisfies the following condition

$$
\varepsilon_{j}(k)>0
$$

and in consequence from (A3) and (A4) it follows that $\eta_{j, k}=1$.

Then, $\Delta V_{j, k}$ along the solutions of $\varepsilon_{j}(k)$ is given by

$$
\Delta V_{j, k}=\underbrace{\max \left\{\begin{array}{c}
-\varepsilon_{j}(k)-W_{j}(k)+\alpha_{1}, \\
\varepsilon_{j}(k)+W_{j}(k)-\alpha_{2}-\mu_{j}, \\
0
\end{array}\right\}}_{V_{j, k+1}}+\underbrace{\min \left\{\begin{array}{c}
\varepsilon_{j}(k)+\mu_{j}-v_{d}-\alpha_{1}, \\
-\varepsilon_{j}(k)+v_{d}+\alpha_{2}, \\
0
\end{array}\right\}}_{-V_{j, k}}, \forall j=1,2 .
$$

From where with help of Assumptions 1 and 2 it can be easily deduced that

$$
\Delta V_{j, k}= \begin{cases}0 & \text { if } \varepsilon_{j}(k) \leq v_{d}+\alpha_{2} \\ -\varepsilon_{j}(k)+v_{d}+\alpha_{2}<0 & \text { if } v_{d}+\alpha_{2}<\varepsilon_{j}(k) \leq \mu_{j}+\alpha_{2}-W_{j}(k) \\ -\mu_{j}+v_{d}+W_{j}(k)<0 & \text { if } \varepsilon_{j}(k)>\mu_{j}+\alpha_{2}-W_{j}(k)\end{cases}
$$

Now, suppose that for $\varepsilon_{j}(k)$ the following condition holds

$$
\varepsilon_{j}(k) \leq 0
$$

and in consequence from (A3) and (A4) it yields that $\eta_{j, k}=0$. Then $\Delta V_{j, k}$ along the solutions of $\varepsilon_{j}(k)$ is given by

$$
\Delta V_{j, k}=\underbrace{\max \left\{\begin{array}{c}
-\varepsilon_{j}(k)-W_{j}(k)+\alpha_{1}-\mu_{j}, \\
\varepsilon_{j}(k)+W_{j}(k)-\alpha_{2}, \\
0
\end{array}\right\}}_{V_{j, k+1}}+\underbrace{\min \left\{\begin{array}{c}
\varepsilon_{j}(k)+\mu_{j}-v_{d}-\alpha_{1}, \\
-\varepsilon_{j}(k)+v_{d}+\alpha_{2}, \\
0
\end{array}\right\}}_{-V_{j, k}}, \forall j=1,2 .
$$

Here with help of Assumptions 1 and 2 it can be easily deduced that

$$
\Delta V_{j, k}= \begin{cases}0 & \text { if } v_{d}+\alpha_{1}-\mu_{j} \leq \varepsilon_{j}(k) \leq 0 \\ -v_{d}-W_{j}(k)<0 & \text { if } \varepsilon_{j}(k)<-\mu_{j}+\alpha_{1}-W_{j}(k), \\ \varepsilon_{j}(k)+\mu_{j}-v_{d}-\alpha_{1}<0 & \text { if }-\mu_{j}+\alpha_{1}+W_{j}(k) \leq \varepsilon_{j}(k)<v_{d}+\alpha_{1}-\mu_{j}\end{cases}
$$

Summarizing, for conditions (A5), (A6), and (A8) from (A7) and (A9) it holds that if $V_{j, k}>0$ its increment $\Delta V_{j, k}<0$. From the definition of min it yields that for $\Delta V_{k}^{2 M}$ given by (A2) the following inequality is satisfied

$$
\Delta V_{k}^{2 M} \leq V_{j, k+1}-V_{j, k} \leq 0
$$


where $i=\arg \max _{j=1,2}\left\{V_{j, k+1}\right\}$. Note that $\Delta V_{j, k}=V_{j, k+1}-V_{j, k}=0$ only if either first condition of (A7) or first condition of (A9) is satisfied and for all $\varepsilon_{j}(k) \notin\left[v_{d}+\alpha_{1}-\right.$ $\left.\mu_{j}, v_{d}+\alpha_{2}\right]$ it follows that $\Delta V_{k}^{2 M}<0$. Thus, in this case it holds that for $V_{k}^{2 M}>0$ given by (A1) its increment $\Delta V_{k}^{2 M}<0$.

Case 2 (Insufficient Buffer Content)

Let us assume that $w_{2}(k)$ satisfies the following inequality

$$
w_{2}(k)<\beta_{2}(k), \forall k \in \mathbb{N}
$$

and $\varepsilon_{2}(k)$ satisfies

$$
\varepsilon_{2}(k) \leq 0, \forall k \in \mathbb{N}
$$

Then from (27) it holds that

$$
\varepsilon_{1}(k)>\mu_{1}+\alpha_{2}-\alpha_{1}+\varepsilon_{2}(k) .
$$

Here similarly to Case 1 the behavior of these two machines can be considered independently. Thus, for $\varepsilon_{1}(k)$ satisfying (A13) it holds that $\Delta V_{1, k}$ is given by (A7) or (A9) if $\varepsilon_{2}(k)<-\mu_{1}-\alpha_{2}+\alpha_{1}$ or $\Delta V_{1, k}$ is given by (A7) if $-\mu_{1}-\alpha_{2}+\alpha_{1} \leq \varepsilon_{2}(k) \leq 0$. For $\varepsilon_{2}(k)$ satisfying (A12) the increment $\Delta V_{2, k}$ is given by (A9). In consequence for $\Delta V_{k}^{2 M}$ given by (A2) the inequality (A10) in this case is also satisfied.

Now, let us assume that $\varepsilon_{2}(k)$ satisfies

$$
\varepsilon_{2}(k)>0, \forall k \in \mathbb{N}
$$

Then from (27) it holds that $\varepsilon_{1}(k)$ is given by (A13). In this case $M_{2}$ has a positive tracking error, but its buffer $B_{2}$ has insufficient raw material content (A11) in order to start working $\left(\eta_{2, k}=0\right)$. Machine $M_{1}$ has a positive error as well, but due to its infinite raw material supply connection it can immediately initiate its production process $\left(\eta_{1, k}=1\right)$. Thus, for (A14) and (A13) let us rewrite $\Delta V_{k}^{2 M}$ from (A2) as

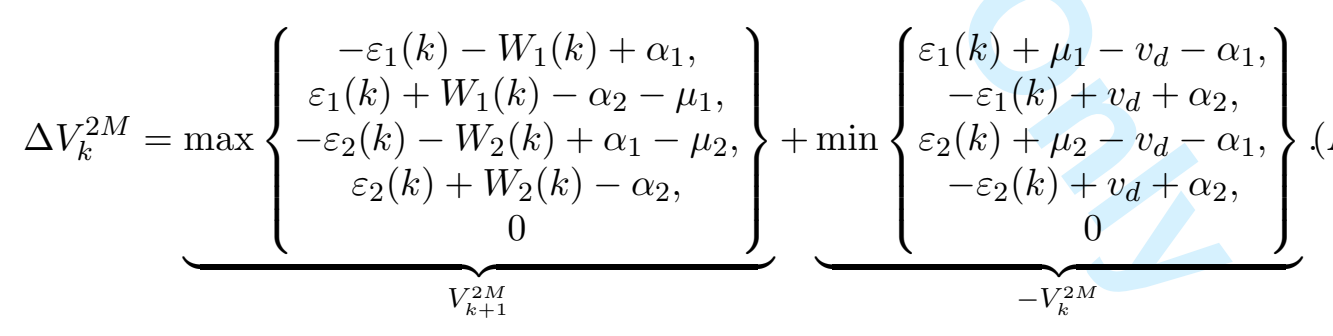

It follows from (20), (A14) and (A13) that $\Delta V_{k}^{2 M}$ from (A15) can be reduced to

$$
\Delta V_{k}^{2 M}=\underbrace{\max \left\{\begin{array}{c}
\varepsilon_{1}(k)+W_{1}(k)-\alpha_{2}-\mu_{1}, \\
\varepsilon_{2}(k)+W_{2}(k)-\alpha_{2}, \\
0
\end{array}\right\}}_{V_{k+1}^{2 M}}+\underbrace{\min \left\{\begin{array}{c}
-\varepsilon_{1}(k)+v_{d}+\alpha_{2}, \\
-\varepsilon_{2}(k)+v_{d}+\alpha_{2}, \\
0
\end{array}\right\}}_{-V_{k}^{2 M}} .
$$


Now, let us prove that for $\varepsilon_{1}(k)$ given by (A13) inequality

$$
\varepsilon_{1}(k)+W_{1}(k)-\alpha_{2}-\mu_{1}>\varepsilon_{2}(k)+W_{2}(k)-\alpha_{2}
$$

is satisfied.

Indeed, from condition (A13) it yields that

$$
\varepsilon_{1}(k)+W_{1}(k)-\alpha_{2}-\mu_{1}>\varepsilon_{2}(k)+W_{1}(k)-\alpha_{1} \stackrel{(20)}{>} \varepsilon_{2}(k)+W_{2}(k)-\alpha_{2} .
$$

Thus, inequality (A17) is satisfied. Also, from (A18) it holds that

$$
\varepsilon_{1}(k)+W_{1}(k)-\alpha_{2}-\mu_{1} \stackrel{(20, A 14)}{>} 0
$$

Now, considering (A17) and (A19) we can rewrite $V_{k+1}^{2 M}$ given by a first term of (A16) as

$$
V_{k+1}^{2 M}=\varepsilon_{1}(k)+W_{1}(k)-\alpha_{2}-\mu_{1} .
$$

Let us prove that for $\varepsilon_{1}(k)$ given by (A13) inequality

$$
-\varepsilon_{2}(k)+v_{d}+\alpha_{2}>-\varepsilon_{1}(k)+v_{d}+\alpha_{2}
$$

is satisfied. Here from condition (A13) it yields that

$$
-\varepsilon_{2}(k)+v_{d}+\alpha_{2}>-\varepsilon_{1}(k)+\mu_{1}+\alpha_{2}-\alpha_{1}+v_{d}+\alpha_{2} \stackrel{(20),(23)}{>}-\varepsilon_{1}(k)+v_{d}+\alpha_{2}(\mathrm{~A} 22)
$$

Thus, inequality (A21) is satisfied. From inequalities (A13), (22) it follows that

$$
-\varepsilon_{1}(k)+v_{d}+\alpha_{2}<0
$$

From (A21), (A23) we can rewrite $V_{k}^{2 M}$ given by a second term of (A16) as

$$
V_{k}^{2 M}=\varepsilon_{1}(k)-v_{d}-\alpha_{2} \stackrel{(A 23)}{>} 0 .
$$

Having $V_{k+1}^{2 M}$ given by (A20) and $V_{k}^{2 M}$ given by (A24), we can finally reduce $\Delta V_{k}^{2 M}$ from (A16) to

$$
\Delta V_{k}^{2 M}=-\mu_{1}+v_{d}+W_{1}(k) \stackrel{(24)}{<} 0 .
$$

Thus, for this Case it holds that for $V_{k}^{2 M}>0$ given by (A1) its increment $\Delta V_{k}^{2 M}<0$. Summarizing for 2 cases, we have shown that for $V_{k}^{2 M}>0$ given by (A1) its increment $\Delta V_{k}^{2 M}<0$ for all $\varepsilon_{j}(k) \notin\left[v_{d}+\alpha_{1}-\mu_{j}, v_{d}+\alpha_{2}\right]$ and $\Delta V_{k}^{2 M}=0$ for all $\varepsilon_{j}(k) \in$ $\left[v_{d}+\alpha_{1}-\mu_{j}, v_{d}+\alpha_{2}\right]$.

Thus, $\limsup _{k \rightarrow \infty} V_{k}^{2 M}=0$ which completes our proof.

In this proof we have analyzed the increment of the proposed Lyapunov function by means of 2 cases. Now for a line of $N$ manufacturing machines $(j=1, \ldots, N)$ defined by 
(18) and (19) the Lyapunov function (A1) is extended to

$$
V_{k}^{N M}=\max \left\{V_{1}\left(\varepsilon_{1}\right), \ldots, V_{N}\left(\varepsilon_{N}\right)\right\}
$$

Here the similar reasoning is followed as for the proof for 2 machines.

The analysis is subdivided into the same 2 cases. Case 1 (Sufficient Buffer Content) and the first part of Case $2\left(w_{j}(k)<\beta_{j}(k)\right.$ and $\left.\varepsilon_{j}(k) \leq 0, \forall j=2, \ldots, N\right)$ are solved identically to the proof for the line of 2 machines. For the second part of Case 2 the proof mostly relies on the condition (27) and the assumption that machine $M_{1}$ has always an access to the infinite raw material supply. Due to the extensive technical details we omit the complete analysis for a line of $N$ machines and restrict ourselves by only giving this general idea of the procedure. 